\title{
The QBIT theory of consciousness
}

\author{
Majid Beshkar \\ Tehran University of Medical Sciences \\ mbeshkar@sina.tums.ac.ir \\ majid.beshkar@gmail.com
}

Abstract

The QBIT theory is an attempt toward solving the problem of consciousness based on empirical evidence provided by various scientific disciplines including quantum mechanics, biology, information theory, and thermodynamics. This theory formulates the problem of consciousness in the following four questions: (1) What is the nature of qualia? (2) How are qualia generated? (3) Why are qualia subjective? (4) Why does a quale have a particular quality or meaning?

In sum, the QBIT theory proposes that (1) when a pack of quantum information is compressed beyond a certain threshold, a quale is generated; (2) a quale is a superdense pack of maximally entangled qubits in a pure state; (3) when information-theoretic certainty of a system about an external stimulus exceeds a particular level, the system becomes conscious of that stimulus; (4) subjectivity of consciousness is due to the fact that maximally entangled pure states are private and unshareable.

Keywords: Coherence; Compression; Computation; Consciousness; Entanglement; Information; Qualia; Quantum; Representation 
Introduction

The problem of consciousness is one of the most difficult problems in biology, which has remained unresolved despite several decades of scientific research. The hard core of the problem of consciousness is in fact the problem of qualia.

Qualia (plural for quale) refers to subjective conscious experiences such as a red color, a sharp pain, a particular smell, or a specific taste. As an example, when we see a red flower, the redness that we experience is a quale. "To be conscious" means "to have qualia", and unconscious perception means "qualia-less perception".

To resolve the problem of consciousness, empirical evidence alone is not sufficient; we also need an appropriate theory to select and put together diverse (and sometimes seemingly unrelated) empirical evidence to reveal a hidden pattern. In this context, the QBIT theory is an attempt toward solving the puzzle of consciousness with pieces of evidence collected from different scientific disciplines including quantum mechanics, biology, information theory, and thermodynamics. In the following sections, basic assumptions and propositions of the QBIT theory are presented and discussed.

"Information compression" is the key to solve the problem of consciousness.

Compression can perform magic. In nature, there is a remarkable example of the magic of compression: extreme compression of matter creates an enigmatic entity called the "black hole". The QBIT theory suggests that extreme compression of information creates another enigmatic entity, a quale. Roughly similar to a black hole (which is a superdense pack of matter), a quale is proposed to be a superdense pack of information. In the terminology of the QBIT theory, "a pack of information" means a representation, signal, a code, or a message. 
The QBIT theory suggests that when a pack of information in a sensory system is compressed beyond a certain threshold, a quale is generated. To achieve such a high level of compression, classical resources are insufficient; quantum resources (including entanglement and coherence) are required. Quantum compression is much more powerful than classical compression. Consistent with this idea, it has been shown that entangled quantum states can be compressed far beyond what is possible via classical compression. ${ }^{1}$

The QBIT theory suggests that there are at least two potential mechanisms by which the brain can compress a pack of information: (1) removing some energy but no information from the pack; and (2) adding some information but no energy to the pack. Therefore, the QBIT theory looks at the concept of "information compression" from an energy perspective rather than from a "length of message" or "code length" perspective. This approach to information compression has been described by Luke Rallan and Vlatko Vedral. ${ }^{2}$ They argue that releasing "redundant energy" (which carries no information) gives rise to information compression. In fact, releasing energy without losing any information corresponds to quantum information compression.

Emergence of consciousness requires iterative hierarchical computation.

As Stanislas Dehaene and his colleagues ${ }^{3}$ nicely argue, although centuries of philosophical dualism have led us to consider consciousness as unreducible to physical interactions, scientific evidence is compatible with the proposition that consciousness arises from nothing more than a particular type of computation. But what is computation, and what kind of computation is required for consciousness? 
In cognitive science, computation could be regarded as transformation of one internal representation into another. ${ }^{4,5}$ Here, "internal representation" is defined as a pack of information within a sensory system that encodes (or represents) an external stimulus. An external stimulus is defined as a pack of energy that activates a sensory receptor.

It is widely accepted in neuroscience and cognitive science that consciousness requires formation and transformation of internal representations by a sensory system. ${ }^{6}$ A sensory system contains a hierarchy of computational nodes. At the lowest level of this hierarchy, there is a sensory receptor (node 1 or N1) that converts the energy of an external stimulus into a pack of information. This pack of information is the lowest-level internal representation (representation 1 or R1) that the system creates to encode the stimulus. This representation (or code) is transmitted up the hierarchy to the next computational node (N2), where the representation undergoes a series of computational operations and, as a consequence, transforms into a higher-level representation(R2). This representation is then transmitted up the hierarchy to the next node (N3), where it is transformed into a representation (R3) that has a higher status than the previous one. This iterative transformation of representations continues until the highest-level representation is created at the top of the hierarchy.

Each computational node (for example, N3) receives at least two packs of information: a bottom-up input which is the representation (or signal) sent forward from the preceding node (N2), and a topdown input which is the signal sent backward from a higher-level computational node (for example, N4). The N3 integrates these packs of information to form a new representation. This new pack of information is compressed by $\mathrm{N} 3$, and the compressed representation is then transmitted to N4 for another round of 
"integration and compression". The highest-level computational node generates the most compressed representation, which is a quale.

A quale is a representation with the least possible entropy.

Satosi Watanabe ${ }^{7}$ argues that many natural phenomena as diverse as pattern recognition in biology and star formation in cosmology could be regarded as the consequences of a universal quest for minimum entropy. The QBIT theory suggests that emergence of consciousness is also a consequence of the quest for minimum entropy.

Joseph Aticks ${ }^{8}$ argues that the visual system is concerned with creating a minimum-entropy representation of an external stimulus. It is very unlikely that any system could achieve this in just one computational step. It is more likely that the system creates such a representation by means of an iterative hierarchical process that gradually reduces entropy.

As a representation ascends the hierarchy, its entropy is gradually reduced. Therefore, the representation generated by the sensory receptor (i.e. R1) has maximum entropy, while the representation generated at the top of the hierarchy has minimum entropy. The QBIT theory suggests that a quale is a representation with the minimum possible entropy.

The entropy of a pack of information determines how much the pack can be compressed. ${ }^{2}$ The less the entropy of a representation, the less compressible it is. A quale is the most compressed representation. It has the least possible entropy, and cannot be compressed further.

A quale is a representation with the maximum mutual information.

As a representation ascends the hierarchy, its mutual information with the external stimulus that it represents is increased. Mutual 
information is in a sense the converse of entropy. ${ }^{9}$ Therefore, the representation generated by the sensory receptor (i.e. R1) has minimal mutual information and maximum entropy, while the representation generated at the top of the hierarchy has maximal mutual information and the least entropy. An idea similar to this has been recently proposed by Daya Shankar Gupta and Andreas Bahmer. ${ }^{10}$ They argue that an increase in mutual information occurs as sensory information is processed successively from lower to higher levels in a cortical hierarchy. They suggest that this gradual increase in mutual information contributes to perception.

Mutual information between two variables ( $\mathrm{X}$ and $\mathrm{Y}$ ) is the average reduction in uncertainty about $X$ that results from knowing the value of $\mathrm{Y}$. Suppose that $\mathrm{X}$ is an external stimulus and $\mathrm{Y}$ is one of its internal representations generated at a computational node of a sensory system. In this context, an increase in mutual information is equivalent to an increase in certainty of the sensory system about the external stimulus. A quale is the highest-level representation, with the least entropy (or uncertainty) and the maximum mutual information.

The QBIT theory suggests that when certainty of a sensory system about an external stimulus exceeds a particular level, the system becomes conscious of that stimulus. To attain such a high level of certainty, quantum information is required. As Patrick Hayden ${ }^{11}$ nicely mentions, "with quantum information, it is possible not just to be certain, but to be more than certain." This wonderful effect of quantum information inspires the idea that in order to become conscious, we need to go beyond the limits of classical physics. Consciousness requires quantum phenomena, including entanglement and coherence. 
A quale is a representation with the maximum predictive accuracy. Predictive coding was first developed as a data compression strategy in signal processing. ${ }^{12}$ It is an encoding strategy by which only unpredicted elements of a signal are transmitted to the next stage for further information processing. ${ }^{13}$ In fact, predictive coding compresses a signal (or a representation) by removing the predictable, and hence redundant, elements of that signal. ${ }^{14}$

In a hierarchical model of predictive coding, as described by Rajesh Rao and Dana Ballard, ${ }^{14}$ a pack of sensory information in a computational node (for example, the primary visual cortex or V1) is compared against a prediction received from a higher-level computational node (for example, V2). As a result of this comparison, deviations from such predictions (called the prediction errors) are identified and only these elements are fed forward to the next computational node. In this context, the prediction error is the difference between a pack of sensory information and a higher-level prediction that both enter a computational node.

In predictive coding, feedback and feedforward connections allow the serial, reciprocal exchange of predictions and prediction errors. ${ }^{15}$ Signals (or packs of information) descending the hierarchy via backward connections (i.e. top-down inputs) contain predictions, while signals ascending the hierarchy via forward connections (i.e. bottom-up inputs) contain prediction errors.

In general, a computational node at any given stage attempts to predict the representation (or the pack of information) generated at the stage below. Furthermore, the same computational node also attempts to improve (or update) the representation at the stage above by reporting its errors of prediction. ${ }^{15}$ As a representation ascends this hierarchy, its errors are gradually minimized. The representation generated at the top of the hierarchy, has the least prediction errors, 
and hence is the most accurate prediction that a sensory system has about the associated external stimulus.

Daniel Little and Friedrich Sommer ${ }^{9}$ argue that the predictive accuracy of an internal representation could be measured by its mutual information with the sensory input. In this context, mutual information is the amount of information a representation contains regarding the associated sensory input. On the basis of these arguments, the QBIT theory suggests that a quale is an internal representation generated at the top of the hierarchy of predictive coding. Therefore, a quale is the most accurate representation, with the least prediction errors, and maximal mutual information.

A quale is the simplest representation of an external stimulus.

The simplicity principle is a powerful unifying principle in cognitive science capable of explaining a wide range of phenomena including perception as well as learning. ${ }^{16}$ The simplicity principle states that a primary goal of sensory processing is to create the simplest possible internal representations of external stimuli. ${ }^{17}$ The tendency of a sensory system to create the simplest possible representations is due to the fact that simplest representations allow the most accurate predictions and provide the best basis for decision-making, both necessary for survival in a challenging environment.

To create the simplest possible representations, a sensory system should be endowed with the capacity to compress information. There is a variety of techniques for information compression that a cognitive system (such as the brain) can exploit to maximize simplicity of its internal representations. One of these techniques is the "matching and unification of patterns" as described by Gerard Wolff. ${ }^{18}$ This kind of information compression is accomplished through a series of computational operations that search a pack of 
information to find patterns that match each other, and then merge or unify them so that multiple configurations of the same pattern are reduced to one. Wolff ${ }^{19}$ argues that compressing a representation (or a pack of information) via the matching and unification of patterns increases both the simplicity and the explanatory power of that representation. He suggests that this kind of information compression via the matching and unification of patterns is an essential part of perception, cognition, and learning in the human brain.

In a sensory system, as a representation ascends the hierarchy, it becomes simpler (or less complex). Therefore, a quale, generated at the top of the hierarchy, is the representation with the least possible complexity. Here, "complexity" is used in the sense of algorithmic (or Kolmogorov) complexity.

Kolmogorov complexity measures the amount of statistical regularity, and not the amount of information, within a representation. ${ }^{20}$ Statistical regularity is a kind of redundancy..${ }^{21}$ Any regular or predictable element of a representation reduces its simplicity. In fact, the degree of simplicity of a representation is inversely related to the amount of statistical regularity it contains. ${ }^{21}$

A quale is a representation with the least possible free energy.

The free-energy principle states that any self-organizing system (such as a sensory system) that is able to resist decay and maintain its integrity over time must constantly minimize its internal entropy by minimizing its variational free energy. ${ }^{22}$ In this context, variational free energy is an information theoretic analogue of the thermodynamic free energy, and entropy is the long-term average of surprisal (or uncertainty). ${ }^{23,24}$ Therefore, minimizing free energy is equivalent to reducing entropy and uncertainty. ${ }^{25}$ Shannon entropy 
(also called uncertainty) quantifies how much is not known about something. ${ }^{26}$ In other words, entropy is a measure of the amount of information needed to eliminate all uncertainty about a variable. ${ }^{27}$

The free-energy principle proposes that adaptive fitness of an organism corresponds to minimization of sensory uncertainty, which is the average of surprisal. ${ }^{28}$ According to this principle, when an organism is stimulated through its sensory receptors, it instantly (and automatically) initiates an attempt to minimize sensory surprisal. ${ }^{28}$

A cognitive system (such as the brain) could minimize its variational free energy by recurrent information passing through a hierarchy of computational nodes, so that each node minimizes uncertainty in the incoming information by receiving a prediction (or a prior) and responding to errors in that prediction. ${ }^{29}$ As a pack of information ascends the hierarchy, its free energy is progressively minimized until a quale is generated.

A quale is the most efficient code that represents an external stimulus.

Efficient coding is a kind of information compression strategy. A computational node (for example, the retina) uses this strategy to compress a pack of information with minimal information loss so that as much information as possible can be transmitted to a higher computational node through a channel of limited capacity (for example, the optic nerve). ${ }^{30}$ Efficient coding often involves removing redundancies from a pack of information. ${ }^{30}$

As a representation ( or code) ascends the hierarchy, it becomes more compressed (i.e., less redundant) and hence more efficient. At the top of the hierarchy, the most efficient code (i.e., a quale) is generated.

A quale is like a chunk. 
In cognitive science, a chunk is defined as a collection of elements having strong associations with one another, but weak or no associations with elements outside the chunk. ${ }^{31}$ Chunking is an information compression strategy used by the brain in perception, cognition, learning, and memory. By this strategy, the brain replaces highly redundant items with a single chunk that represents all of the items. In fact, chunking could be regarded as an example of a universal strategy used by the brain: removal of redundancy to form compressed representations. ${ }^{32}$

The QBIT theory suggests that a quale could be regarded as a kind of chunk. In this context, a quale is a collection of integrated qubits having strong correlation with one another, but no correlation with qubits outside the chunk.

A quale is a superdense pack of quantum information encoded in maximally entangled pure states.

In quantum information theory, local operations and classical communication (LOCC) is a method of information processing in which a local operation is performed in a node of a system, and then the result of that operation is communicated classically to another node where another local operation is performed conditioned on the information received.

The QBIT theory suggests that the iterative hierarchical computation that occurs in a sensory system is, at a more fundamental level, a sequential series of LOCC operations. These operations have important effects on the representation generated at each computational node.

Entanglement distillation and coherence distillation are two important functions of LOCC operations. Entanglement distillation is a process by which a smaller number of maximally entangled 
qubits can be extracted from a large number of less-entangled qubits. ${ }^{33}$ In other words, entanglement distillation can convert a collection of non-maximally entangled qubits to a collection of maximally entangled qubits. Likewise, coherence distillation (also called state purification) is a process by which a collection of qubits in a mixed state can be converted to a collection of qubits in a maximally coherent (i.e. pure) state. ${ }^{34}$ Therefore, a sequential series of LOCC operations can potentially generate maximally entangled pure states. ${ }^{35}$

According to the QBIT theory, a representation generated at any level of the hierarchy is in fact a pack of quantum information or a collection of qubits. As a pack ascends the hierarchy, LOCC operations gradually transforms the pack into a collection of qubits with maximal entanglement and coherence. At the top of the hierarchy, a quale is generated which is a pack of maximally entangled qubits in a pure state.

Maximally entangled pure states are ideal resources for quantum computation, while mixed states are not very useful for this purpose. $^{36}$ Some unique and wonderful effects of quantum computation arise only when maximally entangled pure states are available for use. From a thermodynamic point of view, production of maximally entangled pure states is costly, meaning that it requires consumption of energy and production of entropy. However, for some computational tasks such as estimating a given parameter with a high precision, it is more cost effective for a system to use maximally entangled pure states rather than using already available mixed states. ${ }^{37}$ Therefore, above a certain level of precision, the cost of computation will be reduced if maximally entangled pure states are used.

Quantum entanglement and coherence are real physical resources which are indispensable for certain computational tasks that cannot 
be performed using classical resources such as energy. ${ }^{38,39}$ However, these resources are very fragile at physiologic temperatures as compared to cryogenic temperatures, since the environmental noise increases with increasing temperature, resulting in rapid decoherence and loss of useful entanglement. ${ }^{40} \mathrm{In}$ fact, decoherence is a common obstacle for all phenomena that depends on the capacity of preserving and using quantum coherence and entanglement. ${ }^{41}$

Although coherence and entanglement are so fragile at physiologic temperatures, there is strong evidence that these two quantum phenomena play important roles in certain biological processes, including photosynthesis in plants and bacteria as well as magnetoreception in birds. ${ }^{42-45}$ Furthermore, there is a growing body of literature in support of the idea that entanglement and coherence are also involved in some aspects of cognition. ${ }^{46-49}$

The QBIT theory suggests that quantum entanglement and coherence play an essential role in consciousness. This idea is also the basis of the Orchestrated Objective Reduction (Orch OR) theory of consciousness, developed by Stuart Hameroff and Roger Penrose. ${ }^{50}$ The Orch OR theory suggests that, for consciousness to occur in a system, it is necessary that a sufficient amount of material (e.g. microtubule) be kept in a coherent (or pure) state for a long enough time.

In quantum mechanics, every system has a set of states. A state is called pure if it contains maximal information about the system. ${ }^{51}$ The Orch OR theory suggests that different states of a tubulin represent information in the brain. The theory considers tubulin bits (and quantum bits, or qubits) as entangled coherent states. These coherent (or pure) states of microtubules in one neuron can extend by entanglement to microtubules in adjacent neurons, potentially extending to brain-wide syncytia. ${ }^{50}$ In line with this theory, evidence shows that long-lived quantum coherence is possible in microtubules 
as well as in some other molecules within the brain at physiologic temperatures. ${ }^{52,53}$

Quantum phenomena can explain why qualia are subjective.

A prime feature of qualia is that they are subjective. This means that they are private and unshareable, accessible only to the system that is generating them. Observation or measurement of qualia generated within a system is not possible for any other system.

A quale could be regarded as a private key. In terms of information theory, a private key is a string of bits which has two important features. First, it is perfectly correlated. Second, it is inaccessible to any other person. ${ }^{36}$ The first feature is due to maximal entanglement. The second feature is due to maximal coherence (or purity), because an eavesdropper who attempts to obtain knowledge about the private key will unavoidably disturb it, introducing a phase error into the system, which destroys purity. ${ }^{36}$

"Entanglement is the quantum equivalent of what is meant by privacy." This nice statement, and the argument behind it, in a paper by Ryszard Horodecki and his colleagues ${ }^{36}$ provided insight for the QBIT theory to propose that quantum phenomena might be able to explain the subjectivity of consciousness.

Quantum entanglement has limited shareability. In the case of pure states, it can even be absolutely unshareable. ${ }^{54}$ All these arguments can be expressed in terms of the monogamy of entanglement. According to the monogamy of entanglement, maximally entangled pure states are not shareable. ${ }^{55,56}$ Since qualia are encoded in maximally entangled pure states, they should be private and unshareable. 
The meaning of a quale is assigned by the iterative hierarchical computation.

Information on its own has no intrinsic meaning. It is "interpretation" that adds a meaning to information. The same pack of information can have different meanings, depending on how it is interpreted by a system. ${ }^{57}$ The QBIT theory suggests that, in a sensory system, what interprets a pack of information and assigns a particular meaning to it is the iterative hierarchical computation. Computations performed at each stage of the hierarchy could be regarded as a kind of "interpretation" that gives a particular meaning to the representation before being sent to the next stage. As a representation ascends the hierarchy, it becomes not only more compressed but also more meaningful for the system. Consistent with this conjecture, Stephan Tschechne and Heiko Neumann ${ }^{58}$ argue that computations in early and intermediate stages of visual hierarchy transform local representations into more meaningful representations of contours, shapes and surfaces.

\section{Final remarks}

The QBIT theory proposes that qualia are quantum information in nature, and emergence of qualia requires quantum computation. Most physical phenomena in nature can be formulated and better described in terms of quantum information and computation. 59 Gravity is a prominent example. Reconciling quantum mechanics with gravity is a hard and yet unresolved problem in physics. Recently, quantum information theory and concepts like entanglement and quantum error correction have come to play a fundamental role in solving this problem. For example, it has been suggested that gravity comes from quantum information. ${ }^{60}$ Furthermore, recent evidence from theoretical physics imply that entangled qubits are not only the origin of gravity, but also the origin 
of matter and space. ${ }^{61}$ It seems that, at some level, everything reduces to information. ${ }^{62}$ This inspires the QBIT theory to propose that, at a fundamental level, qualia are quantum information or entangled qubits.

As our knowledge about the nature of quantum information increases, we would gain more insights about the nature of qualia. At present, we know that quantum information is nonlocal. It does not make sense to ask where quantum information is at any given time; it is nonlocally distributed in the entangled state. ${ }^{56}$ Since quantum information is nonlocal, qualia should also be nonlocal. Furthermore, there is some evidence that quantum information is physical. ${ }^{63}$ If this turns out to be true, then qualia must also be physical.

In general, information cannot exist without a physical substrate that encodes it. ${ }^{64}$ Therefore, information that we retain in our brains should also have a physical substrate. This physical substrate is a kind of qubit. But what plays the role of qubits in the brain? There are, at least, two potential candidates: the "tubulin bits" described by Stuart Hameroff and Roger Penrose, ${ }^{50}$ and the "neural qubits" described by the physicist Matthew Fisher. ${ }^{65}$

Matthew Fisher ${ }^{65}$ suggests that, in the brain, nuclear spin of a single phosphorus atom residing on a Posner molecule can serve as a qubit, called a "neural qubit". A Posner molecule is a kind of calcium phosphate molecule with a unique chemical structure that can protect phosphorus nuclear spins from decoherence for very long times. Phosphorus nuclear spins in different Posner molecules can become entangled and remain so for relatively long periods of time. ${ }^{53}$ In the brain, the Posner molecule seems to be a promising platform for quantum computations based on phosphorus nuclear spin. The nucleus of a phosphorus atom is an extremely weak magnet. It can be thought of as a compass needle that can point toward either north 
or south. These north or south positions are equivalent to zeros and ones of binary codes which form the basis of classical computation. In classical computers, information is encoded in zeros and ones, which themselves are represented by different voltages on semiconductors. ${ }^{66}$

\section{Conclusion}

The QBIT theory formulates the problem of consciousness in the following four questions, and provide a preliminary answer for each question:

Question 1: What is the nature of qualia? Answer: A quale is a superdense pack of quantum information encoded in maximally entangled pure states.

Question 2: How are qualia generated? Answer: When a pack of quantum information in a sensory system is compressed beyond a certain threshold, a quale is generated.

Question 3: Why are qualia subjective? Answer: A quale is subjective because a pack of information encoded in maximally entangled pure states are essentially private and unshareable.

Question 4: Why does a quale have a particular meaning? Answer: A pack of information within a cognitive system gradually obtains a particular meaning as it undergoes a progressive process of interpretation performed by a generative model installed in the system.

The QBIT theory of consciousness is in its first stage of development, attempting to absorb relevant evidence from various scientific disciplines. Apparently, it is not a complete and comprehensive theory, but I think it is on the right path toward solving the problem of consciousness. 


\section{References}

1. Reif JH, Chakraborty S. (2007) Efficient and exact quantum compression. Information and Computation, 205: 967-981.

2. Rallan L, Vedral V. (2003) Energy requirements for quantum data compression and 1-1 coding. Physical Review A, 68: 042309.

3. Dehaene S, Lau H, Kouider S. (2017) What is consciousness, and could machines have it? Science, 358(6362):486-492.

4. Sanger TD. (2003) Neural population codes. Current Opinion in Neurobiology, 13: 238-249.

5. Eliasmith C. (2010) How we ought to describe computation in the brain. Studies in History and Philosophy of Science, 41: 313-320.

6. Pennartz CMA. (2018) Consciousness, Representation, Action: The Importance of Being Goal-Directed. Trends in Cognitive Sciences, 22(2): 137-153.

7. Watanabe S. (1981) Pattern recognition as a quest for minimum entropy. Pattern Recognition, 13(5): 381-387.

8. Aticks JJ. (2011) Could information theory provide an ecological theory of sensory processing? Network: Computation in Neural Systems, 22(1-4):4-44.

9. Little DY, Sommer FT. (2013) Maximal mutual information, not minimal entropy, for escaping the "Dark Room". Behavioral and Brain Sciences, 36(3): 220-221.

10. Gupta DS, Bahmer A. (2019) Increase in Mutual Information During Interaction with the Environment Contributes to Perception. Entropy, 21: 365.

11. Hayden P. (2005) Quantum information: putting certainty in the bank. Nature, 436(7051): 633-634. 
12. Clark A. (2013) Whatever next? Predictive brains, situated agents, and the future of cognitive science. Behavioral and Brain Sciences, 36(3): 181-204.

13. Williams D. (2018) Predictive coding and thought. Synthese, DOI: $10.1007 / \mathrm{s} 11229-018-1768-\mathrm{x}$.

14. Rao RPN, Ballard DH. (1999) Predictive coding in the visual cortex: a functional interpretation of some extra-classical receptive-field effects. Nature Neuroscience, 2(1): 79-87.

15. Shipp S. (2016) Neural Elements for Predictive Coding. Frontiers in Psychology, 7: 1792.

16. Chater N, Vitanyi P. (2003) Simplicity: a unifying principle in cognitive science? Trends in Cognitive Sciences, 7(1): 1922.

17. Chater N. (1999) The Search for Simplicity: A Fundamental Cognitive Principle? Quarterly Journal of Experimental Psychology, 52A(2): 273-302.

18. Wolff JG. (2016) Information Compression, Multiple Alignment, and the Representation and Processing of Knowledge in the Brain. Frontiers in Psychology, 7:1584.

19. Wolff JG. (2019) Information Compression as a Unifying Principle in Human Learning, Perception, and Cognition. Complexity, 2019: 1879746.

20. Adami C. (2002) What is complexity? BioEssays, 24: 10851094.

21. Barlow HB. (1974) Inductive inference, coding, perception, and language. Perception, 3: 123-134.

22. Friston K. (2010) The free-energy principle: a unified brain theory? Nature Reviews Neuroscience, 11: 127-138.

23. Kirchhoff M, Parr T, Palacios E, Friston K, Kiverstein J. (2018) The Markov blankets of life: autonomy, active inference and the free energy principle. Journal of the Royal Society Interface, 15: 20170792. 
24. Ramstead MJD, Badcock PB, Friston KJ. (2018) Answering Schrödinger's question: A free-energy formulation. Physics of Life Review, 24: 1-16.

25. Kirchhoff MD, Froese T. (2017) Where There Is Life There Is Mind: In Support of a Strong Life-Mind Continuity Thesis. Entropy, 19: 169.

26. Adami C. (2016) What is information? Philosophical Transactions of the Royal Society A, 374: 20150230. DOI: $10.1098 /$ rsta.2015.0230

27. Borst A, Theunissen FE. (1999) Information theory and neural coding. Nature Neuroscience, 2(11): 947-957.

28. Kim CS. (2018) Recognition Dynamics in the Brain under the Free Energy Principle. Neural Computation, 30: 26162659 .

29. Fotopoulou A. (2013) Beyond the Reward Principle: Consciousness as Precision Seeking. Neuropsychoanalysis, 15(1):33-38.

30. Zhaoping L. (2006) Theoretical understanding of the early visual processes by data compression and data selection. Network: Computation in Neural Systems, 17(4): 301-334.

31. Gobet F, Lane PCR, Croker S, Cheng PCH, Jones G, Oliver I, Pine JM. (2001) Chunking mechanisms in human learning. Trends in Cognitive Sciences, 5(6): 236-243.

32. Brady TF, Konkle T, Alvarez GA. (2009) Compression in Visual Working Memory: Using Statistical Regularities to Form More Efficient Memory Representations. Journal of Experimental Psychology: General, 138(4): 487-502.

33. Pan J-W, Gasparoni S, Ursin R, Weihs G, Zeilinger A. (2003) Experimental entanglement purification of arbitrary unknown states. Nature, 423(6938): 417- 422.

34. Liu CL, Zhou DL. (2019) Deterministic Coherence Distillation. Physical Review Letters, 123: 070402. 
35. Murao M, Vedral V. (2001) Remote Information Concentration Using a Bound Entangled State. Physical Review Letters, 86(2): 352-355.

36. Horodecki R, Horodecki P, Horodecki M, Horodecki K. (2009) Quantum entanglement. Reviews of Modern Physics, 81(2): 865-942.

37. Cirac JI, Ekert AK, Huelga SF, Macchiavello C. (1999) Distributed quantum computation over noisy channels. Physical Review A, 59: 4249.

38. Maruyama K, Morikoshi F, Vedral V. (2005) Thermodynamical detection of entanglement by Maxwell's demons. Physical Review A, 71: 012108.

39. Streltsov A, Adesso G, Plenio MB. (2017) Colloquium: Quantum coherence as a resource. Reviews of Modern Physics, 89: 041003.

40. Marais A, Adams B, Ringsmuth AK, et al. (2018) The future of quantum biology. Journal of the Royal Society Interface, 15: 20180640.

41. Viola L, Knill E, Lloyd S. (1999) Dynamical Decoupling of Open Quantum Systems. Physical Review Letters, 82: 24172421.

42. Engel GS, Calhoun TR, Read EL, Ahn TK, Mancal T, Cheng YC, Blankenship RE, Fleming GR. (2007) Evidence for wavelike energy transfer through quantum coherence in photosynthetic systems. Nature, 446(7137): 782-786.

43. Gauger EM, Rieper E, Morton JJ, Benjamin SC, Vedral V. (2011) Sustained quantum coherence and entanglement in the avian compass. Physical Review Letters, 106(4): 040503.

44. Lambert N, Chen Y-N, Cheng Y-C, Li C-M, Chen G-Y, Nori F. (2013) Quantum biology. Nature Physics, 9: 10-18. 
45. Brookes JC. (2017) Quantum effects in biology: golden rule in enzymes, olfaction, photosynthesis and magnetodetection. Proceedings of the Royal Society A, 473: 20160822.

46. Wang Z, Busemeyer JR, Atmanspacher H, Pothos EM. (2013) The potential of using quantum theory to build models of cognition. Topics in Cognitive Science, 5: 672-688.

47. Hameroff S. (2014) Quantum Walks in Brain Microtubules-A Biomolecular Basis for Quantum Cognition? Topics in Cognitive Science, 6: 91-97.

48. Busemeyer JR, Wang Z. (2015) What is quantum cognition, and how is it applied to psychology? Current Directions in Psychological Science, 24: 163- 169.

49. Surov IA, Pilkevich SV, Alodjants AP, Khmelevsky SV. (2019) Quantum Phase Stability in Human Cognition. Frontiers in Psychology, 10: 929.

50. Hameroff S, Penrose R. (2014) Consciousness in the universe: A review of the 'Orch OR' theory. Physics of Life Reviews, 11(1): 39-78.

51. Atmanspacher H, Römer H, Walach H. (2002) Weak Quantum Theory: Complementarity and Entanglement in Physics and Beyond. Foundations of Physics, 32(3):379-406.

52. Craddock TJA, Friesen D, Mane J, Hameroff S, Tuszynski JA. (2014) The feasibility of coherent energy transfer in microtubules. Journal of the Royal Society Interface, 11: 20140677.

53. Weingarten CP, Doraiswamy PM, Fisher MPA. (2016) A New Spin on Neural Processing: Quantum Cognition. Frontiers in Human Neuroscience, 10: 541.

54. Seevinck MP. (2010) Monogamy of correlations versus monogamy of entanglement. Quantum Information Processing, 9(2): 273-294. 
55. Doherty AC. (2014) Entanglement and the shareability of quantum states. Journal of Physics A: Mathematical and Theoretical, 47: 424004.

56. Susskind L, Zhao Y. (2018) Teleportation through the wormhole. Physical Review D, 98: 046016.

57. Orpwood R. (2007) Neurobiological mechanisms underlying qualia. Journal of Integrative Neuroscience, 6(4):523-540.

58. Tschechne S, Neumann H. (2014) Hierarchical representation of shapes in visual cortex-from localized features to figural shape segregation. Frontiers in Computational Neuroscience, 8: 93.

59. Luo S. (2003) Wigner-Yanase Skew Information and Uncertainty Relations. Physical Review Letters, 91: 180403.

60. Qi XL. (2018) Does gravity come from quantum information? Nature Physics, 14: 984-987.

61. Wen X-G. (2019) Choreographed entanglement dances: Topological states of quantum matter. Science, 363(6429): eaal3099.

62. Masanes L, Müller MP, Augusiak R, Pérez-García D. (2013) Existence of an information unit as a postulate of quantum theory. Proceedings of the National Academy of Sciences USA, 110(41): 16373-16377.

63. DiVincenzo DP, Loss D. (1998) Quantum information is physical. Superlattices and Microstructures, 23(3-4): 419-432.

64. Landauer R. (1991) Information is physical. Physics Today, 44: 23-29.

65. Fisher MPA. (2015) Quantum cognition: the possibility of processing with nuclear spins in the brain. Annals of Physics, 362: 593-602.

66. Adami C. (2012) The use of information theory in evolutionary biology. Annals of the New York Academy of Sciences, 1256(2012): 49-65. 